\title{
Vitamin D and Hashimoto's Thyroiditis: Observations from CROHT Biobank
}

\author{
Maja Cvek ${ }^{1}$, Dean Kaličanin ${ }^{2} \mathbb{D}$, Ana Barić ${ }^{1}$, Marko Vuletić ${ }^{1}$, Ivana Gunjača ${ }^{2} \mathbb{D}$, Vesela Torlak Lovrić ${ }^{1}$, \\ Veselin Škrabić ${ }^{3}$, Ante Punda ${ }^{1}$ and Vesna Boraska Perica ${ }^{2, *(D)}$ \\ 1 Department of Nuclear Medicine, University Hospital of Split, 21000 Split, Croatia; \\ maja.cvek.st@gmail.com (M.C.); ana.baaric@gmail.com (A.B.); mavuletic@gmail.com (M.V.); \\ veselakbsplit@yahoo.com (V.T.L.); ante.punda@gmail.com (A.P.) \\ 2 Department of Medical Biology, School of Medicine, University of Split, 21000 Split, Croatia; \\ dkalican@mefst.hr (D.K.); igunjaca@mefst.hr (I.G.) \\ 3 Department of Pediatrics, University Hospital of Split, 21000 Split, Croatia; vskrabic@kbsplit.hr \\ * Correspondence: vboraska@mefst.hr; Tel.: +385-91-534-15-12
}

check for

updates

Citation: Cvek, M.; Kaličanin, D.;

Barić, A.; Vuletić, M.; Gunjača, I.;

Torlak Lovrić, V.; Škrabić, V.; Punda,

A.; Boraska Perica, V. Vitamin D and

Hashimoto's Thyroiditis:

Observations from CROHT Biobank.

Nutrients 2021, 13, 2793.

https://doi.org/10.3390/

nu13082793

Academic Editor: John H. White

Received: 29 July 2021

Accepted: 13 August 2021

Published: 15 August 2021

Publisher's Note: MDPI stays neutra with regard to jurisdictional claims in published maps and institutional affiliations.

Copyright: (c) 2021 by the authors. Licensee MDPI, Basel, Switzerland. This article is an open access article distributed under the terms and conditions of the Creative Commons Attribution (CC BY) license (https:// creativecommons.org/licenses/by/ $4.0 /$ )

\begin{abstract}
The aims of this study were to evaluate: (1) associations of vitamin D with the presence/severity of Hashimoto's thyroiditis (HT) and (2) correlations of vitamin D with thyroid-related phenotypes. Total 25(OH)D (vitamin D in the text) was measured from stored serum samples of 461 HT patients and 176 controls from a Croatian Biobank of HT patients (CROHT). (1) Vitamin D levels, and proportions of vitamin D deficiency, were compared between HT cases and controls. HT patients were additionally divided into two groups (MILD and OVERT) to take into account HT severity. (2) Correlations between vitamin D and 10 clinical phenotypes in all HT patients and two subgroups of HT patients were tested using the Spearman correlation test. Our analyses were adjusted for age, gender, BMI, smoking status and seasonality of blood sampling. (1) No significant differences in vitamin D levels, or proportions of vitamin D deficiency, were detected between HT patients of all disease stages and controls. However, a nominally significant difference in vitamin D levels between MILD and OVERT subgroups (OR $=1.038, p=0.023)$ was observed. Proportions of individuals with vitamin D deficiency during winter-spring were high: all HT cases (64.69\%), MILD (60.64\%), OVERT (68.7\%), controls (60.79\%). (2) A nominally significant negative correlation between vitamin D and TSH in all HT patients $(r=-0.113, p=0.029)$ and a positive correlation between vitamin D and systolic blood pressure in OVERT HT patients $(r=0.205, p=0.025)$ were identified. Our study indicates that there is no association between vitamin D and HT; however, there may be a subtle decrease in vitamin D levels associated with overt hypothyroidism.
\end{abstract}

Keywords: vitamin D; Hashimoto's thyroiditis; thyroid autoimmunity; thyroid stimulating hormone (TSH); thyrotropin

\section{Introduction}

Vitamin D, traditionally known as a fat-soluble vitamin, is a confirmed steroid hormone with its main role in calcium and phosphorus homeostasis and the regulation of bone metabolism. The term "vitamin D" stands for two compounds: cholecalciferol (vitamin D3) and ergocalciferol (vitamin D2) [1]. Vitamin D3 is synthesized in the skin (about 80\%) by sunlight exposure (UVB radiation) while vitamin D2 is produced by plants, fungi and yeast (about 20\%) [1,2]. Humans get vitamin D from three different sources: skin production, diet and supplementation.

Both forms of vitamin $\mathrm{D}$ are hydroxylated to 25-hydroxyvitamin $\mathrm{D}, 25(\mathrm{OH}) \mathrm{D}$ or calcidiol, which is the major circulating form of vitamin D with a half-life of 2-3 weeks [3]. $25(\mathrm{OH}) \mathrm{D}$ is measured and used as an indicator of the vitamin D status in an organism. The first hydroxylation takes place in the liver, while the second one occurs in the kidney by the action of 1- $\alpha$-hydroxylase, CYP27B1, where calcidiol is hydroxylated to calcitriol 
$(1,25(\mathrm{OH}) 2 \mathrm{D})$, the biologically active form of vitamin $\mathrm{D}[4] .1,25(\mathrm{OH}) 2 \mathrm{D}$ binds to the nuclear vitamin D receptor (VDR) in target tissues [5] thus regulating the expression of more than 200 genes (3-5\% of the human genome) [6]. VDR and the enzyme CYP27B1 are not only found in the small intestine, skeletal and renal cells, as was thought earlier, but in almost all body cells, including thyroid and immune cells [7].

Sufficient levels of vitamin D are important for human health. The role of vitamin D is shown in a variety of endocrine and autoimmune diseases (type 1 diabetes, type 2 diabetes, polycystic ovary syndrome, adrenal diseases, multiple sclerosis, systemic lupus erythematosus, rheumatoid arthritis, AITD), cancers, inflammatory responses, infectious and cardiovascular diseases [8,9]. According to the Endocrine Society Guidelines, a sufficiency of vitamin $\mathrm{D}$ is considered when serum $25(\mathrm{OH}) \mathrm{D}$ is equal to or greater than $30 \mathrm{ng} / \mathrm{mL}$ and up to $100 \mathrm{ng} / \mathrm{mL}$ ( $\geq 75 \mathrm{nmol} / \mathrm{L}$ up to $250 \mathrm{nmol} / \mathrm{L}$ ), insufficiency is considered when vitamin D levels are between 20 and $29.9 \mathrm{ng} / \mathrm{mL}$, while vitamin D deficiency is considered when serum $25(\mathrm{OH}) \mathrm{D}$ is below $20 \mathrm{ng} / \mathrm{mL}$ [10]. The worldwide prevalence of vitamin $\mathrm{D}$ deficiency is high [11,12] and for Europeans it is estimated to be about $40 \%$ [13].

Hashimoto's thyroiditis (HT), chronic lymphocytic or autoimmune thyroiditis, is one of the thyroid diseases with a prevalence of about $10-12 \%$ and an increasing incidence, and is considered to be the most frequent thyroid disorder worldwide [14,15]. Age, gender and race are known HT modifiers, thus white women from 45 to 55 years of age, are four to ten times more affected by HT than males [16]. Main HT characteristics are lymphocyte infiltration into the thyroid gland, causing progressive tissue destruction and the production of antithyroid antibodies, thyroid peroxidase antibodies (TPOAb) and thyroglobulin antibodies (TgAb) [17]. Thyroid autoantibodies are, together with thyroid ultrasound, characteristic features and useful markers for HT diagnosis [18]. The clinical manifestation of disease ranges from mild, characterized by the presence of either one or both types of thyroid antibodies in euthyroid patients, to the gradual development to subclinical and overt hypothyroidism, with or without goiter [18]. HT is caused by the combined action of genetic (about $70 \%$ ), environmental (20-30\%) and existential factors [19]. The most known environmental risk factor for HT is iodine excess, while other suggested ones are, infections (viral and bacterial), medications (e.g., interferon $\alpha$, amiodarone), and chemicals (e.g., polyaromatic hydrocarbons or polyhalogenated biphenyls) [20-23]. In the focus of scientific investigations, as potential environmental factors, there are vitamin $\mathrm{D}$, selenium and gluten [24-26], as well as dietary habits. More work is needed to identify the impact of environmental factors on disease appearance and/or its progression.

The (patho)physiological mechanism of vitamin D involvement in HT development has not been clearly explained yet. The first report about the relationship between vitamin $\mathrm{D}$ and thyroid autoimmunity was published in 2009 [27] when Goswami et al. showed an inverse correlation between 25(OH)D and TPOAb in 642 adults (teachers, students, staff). Since then, a large number of studies tried to clarify the association of vitamin D with HT by analyzing differences in vitamin D levels between HT cases and control participants, but without reaching consistent results. The most recent study found lower vitamin D levels in 373 HT cases in comparison to 4889 control participants who had vitamin $\mathrm{D}$ measured at the same time; however, after performing multivariate logistic regression the study concluded that vitamin D was not associated with HT [28]. Another recent systematic review, meta-analysis and meta-regression of observational studies [29] reported a significant association between vitamin D status and HT, suggesting that HT patients have lower serum $25(\mathrm{OH}) \mathrm{D}$ concentrations than those without HT. This metaanalysis investigated and summarized data across twenty-five studies consisting of 2695 cases and 2263 control individuals. However, the majority of studies were of a small sample size, and only four of them had more than 200 subjects in the HT group. More importantly, many studies that have not found an association between vitamin D and HT were not incorporated in this meta-analysis [30-33]. The weakness of these studies is the limited number of participants. 
Given the inconclusive results on the relationship between vitamin D and HT, we aimed to perform a comprehensive set of analyses, in a large group of clinically diagnosed HT patients and control individuals from the Croatian Biobank of HT patients (CROHT biobank) $[34,35]$ to give more conclusive results on this topic. A recent systematic review of vitamin D concentrations in relation to HT [29] pointed to several moderators that contribute to patient heterogeneity. Besides known factors that can modify vitamin D levels, such as age [36], sex [37], BMI [38] and seasonality [39-41], there are many others that are usually overlooked when studying vitamin D levels, such as smoking, severity of illness, outdoor activity, education, occupation or income [29]. In our study, we used all available information from the CROHT biobank to extrapolate as many of these potential "vitamin D modifying factors" as possible. We therefore used adjustments for age, sex, BMI, smoking and seasonality to minimize the impact of these factors on patient vitamin D levels. We also stratified our HT cases according to HT severity and performed sub-analyses in these groups to take into account the severity of illness. Taken altogether, our study has several aims: the first one is to determine if vitamin D levels differ between HT cases (and HT cases stratified by severity) and controls, the second one is to evaluate the proportions of vitamin D deficiency in study participants, and the final one is to extensively evaluate correlations between vitamin D levels and thyroid-specific phenotypes in HT patients and the subgroups of patients according to illness severity.

\section{Materials and Methods}

Subjects: This is a retrospective observational study in which we used stored serum samples for vitamin D measurements, and phenotype information from HT patients and control participants from the CROHT biobank $[34,35]$. Total $25(\mathrm{OH}) \mathrm{D}$ (vitamin $\mathrm{D}$ in the text) was measured in $461 \mathrm{HT}$ patients $(92.41 \%$ females) and 176 control participants $(93.75 \%$ females) by the LIAISON 25(OH) Vitamin D Total chemiluminescence immunoassay (DiaSorin, Saluggia, Italy). All study subjects from the CROHT biobank were over the age of 18 and during the recruitment period were examined by clinical specialists in nuclear medicine at the Outpatient clinic for thyroid disorders in the Clinical Department of Nuclear Medicine at the University Hospital of Split. HT patients were recruited from 2015 to 2017, covering all seasons, whereas control participants were recruited from December 2018 till June 2019, covering winter and spring. All subjects that used medications and dietary supplements that may impact vitamin D levels (corticosteroids, anticonvulsants, vitamin D supplementation or daily use of calcium supplementation), were excluded from this study.

Diagnosis of HT was made following the ETA recommendations and guidelines for the Management of Subclinical Hypothyroidism [42]. The inclusion criteria for HT patients were: (1) echographic pattern of diffuse thyroid disease; (2) increased thyroid-stimulating hormone (TSH) and/or decreased thyroid hormones-triiodothyronine (T3), thyroxine (T4) or free thyroxine (fT4) and/or increased thyroid autoantibodies (TPOAb and TgAb). The absence of HT in control participants was also established on the basis of clinical examination, following these criteria: (1) echographic pattern of homogenous, normoechogenic (isoechogenic) thyroid parenchyma, with an absence of any diffuse or focal lesions; (2) TSH, T3, T4, fT4, TPOAb and TgAb within reference ranges: TSH (0.3-3.6 mIU/L), T3 (1.3-3.6 nmol/L), T4 (57.3-161 nmol/L), fT4 (10.3-22.8 pmol/L), TPOAb (1-16 IU/mL), $\operatorname{TgAb}(5-100 \mathrm{IU} / \mathrm{mL})$.

Multiple phenotypes were directly measured in study participants or collected using a questionnaires during their enrolment in the CROHT biobank: thyroid related traits (TSH, T3, T4, fT4, TPOAb, TgAb, thyroid gland ultrasound, levothyroxine (LT4) intake, presence/absence of the 16 most common symptoms of hypothyroidism), information on personal anamnesis, comorbidities, main anthropometric and cardiovascular features, information on medical treatments, use of drugs, smoking status, physical activity, dietary habits and many others. During the recruitment stage, blood samples were collected from each participant and used for the measurement of thyroid hormones and antibodies 
(TSH, T3, T4, fT4, TgAb and TPOAb) using LIAISON chemiluminescence immunoassays (DiaSorin Saluggia, Italy). The remaining serum/plasma was stored in a freezer at $-80^{\circ} \mathrm{C}$. The thyroid volume was calculated using dimensions obtained by thyroid gland ultrasound, as a sum of the volumes of both lobes of the thyroid gland. The volume of each lobe was calculated as length $\times$ width $\times$ depth $\times 0.479$ [43]. The main clinical features of study participants are summarized in Table 1.

Table 1. Clinical characteristics of HT patients and control participants.

\begin{tabular}{|c|c|c|c|c|}
\hline \multicolumn{5}{|c|}{ HT Patients } \\
\hline \multirow{3}{*}{ Phenotype } & ALL & MILD & OVERT & Controls \\
\hline & $(N=461)$ & $(\mathrm{N}=240)$ & $(N=219)$ & $(N=176)$ \\
\hline & Median (Q1-Q3) & Median (Q1-Q3) & Median (Q1-Q3) & Median (Q1-Q3) \\
\hline Age, years & $38.02(27.76-48.49)$ & $35.81(25.78-46.95)$ & $40.28(31.04-50.37)$ & $35.17(30.12-44.31)$ \\
\hline BMI, $\mathrm{kg} / \mathrm{m}^{2}$ & $23.52(20.76-26.85)$ & $23.15(20.72-26.59)$ & $24.01(21.02-26.99)$ & $22.66(20.96-25.24)$ \\
\hline $\mathrm{TSH}, \mathrm{mIU} / \mathrm{L}$ & $3.33(1.74-5.68)$ & $3.23(1.82-4.74)$ & $3.52(1.67-12.30)$ & $1.48(1.14-1.95)$ \\
\hline $\mathrm{T} 3, \mathrm{nmol} / \mathrm{L}$ & $1.60(1.30-1.80)$ & $1.70(1.50-1.90)$ & $1.50(1.20-1.80)$ & $1.50(1.40-1.70)$ \\
\hline $\mathrm{T} 4, \mathrm{nmol} / \mathrm{L}$ & $105(89-118)$ & $106(91-117.25)$ & $103(84.85-121)$ & $101(89.37-116)$ \\
\hline $\mathrm{fT} 4, \mathrm{pmol} / \mathrm{L}$ & $12.10(10.20-13.20)$ & $12.10(10.90-13.10)$ & $11.90(9.90-13.70)$ & $12.70(11.90-13.70)$ \\
\hline $\mathrm{TgAb}, \mathrm{IU} / \mathrm{ml}$ & $135(36.40-422.40)$ & $121.50(26.40-321.30)$ & $192(49.30-596.05)$ & $10.75(9.10-18.00)$ \\
\hline TPOAb, IU/ml & $212(27.60-652.90)$ & $161.50(17.40-529.75)$ & $273(66.40-945.50)$ & $3.40(1.20-8.70)$ \\
\hline Thyroid volume, $\mathrm{cm}^{3}$ & $9.85(7.30-13.91)$ & $9.89(7.72-13.26)$ & $9.59(6.82-14.90)$ & $8.69(6.63-10.60)$ \\
\hline No. of symptoms & $4(1-7)$ & $3(1-6)$ & $5(2-8)$ & / \\
\hline Systolic bp, mmHg & $120(110-130)$ & $115(110-130)$ & $120(110-130)$ & $110(100-120)$ \\
\hline Diastolic bp, $\mathrm{mmHg}$ & $70(65-80)$ & $70(65-78.75)$ & $70(65-80)$ & $65(60-80)$ \\
\hline
\end{tabular}

Q1-first quartile, Q3-third quartile; two HT patients were not reallocated in the MILD/OVERT group.

All study participants signed an Agreement for participation prior to inclusion in the study. Two Ethics Committees, one from the University of Split School of Medicine (Classification no. 003-08/14-03/0001 and Registry no. 2181-198-03-04-14-0028; Classification no. 003-08/19-03/0003 and Reg. no. 2181-198-03-04-19-0019) and the other from the University Hospital of Split (Classification no. 530-02/13-01/11; Registry no. 2181-147-01/06/J.B.-14-2; Classification no. 500-03/18-01/80 and Reg. no. 2181-147-01/06/M.S.-18-2) approved this research and declared that it was in accordance with the provisions of the Code of Ethics and the Helsinki Declaration.

Statistical analyses: A sample size was calculated prior to performing statistical analyzes (available online: https://www.stat.ubc.ca/ rollin/stats/ssize/ (accessed on 11 August 2021)). In order to achieve a study power of $80 \%$ with a type I error rate of 0.05 , the number of samples for each group should be at least 121 individuals. To analyze differences in vitamin D levels between $461 \mathrm{HT}$ patients and 175 controls, a logistic regression model was used, where case/control status was used as the dependent variable and vitamin D as an independent variable, along with age, gender, BMI, smoking status and seasonality of blood sampling (a seasonality of sampling was missing for one control, therefore, it was omitted from this analysis). An identical analysis using a subset of $313 \mathrm{HT}$ patients that were recruited during the same time span as control individuals, covering winter and spring, was additionally performed.

In the subsequent analysis, our HT patients were classified into two subgroups depending on HT severity at the time of recruitment. The first group, MILD, incorporated $240 \mathrm{HT}$ patients that were euthyroid (TSH within reference ranges) or in subclinical hypothyroidism (TSH within the range of 3.6-10 mIU/L). The second group, OVERT, contained 219 patients that were in overt hypothyroidism $(\mathrm{TSH}>10 \mathrm{mIU} / \mathrm{L})$ or were treated with levothyroxine (LT4) therapy due to previously established overt hypothyroidism (two HT patients were not reallocated in these two groups due to missing data on their therapy status). Differences in vitamin D levels were compared between each of the two subgroups of HT patients with control participants and additionally using a subset of MILD (155) 
and OVERT (147) patients that matched the controls for the season of the blood draw. Additionally, an in-between comparison of the two subgroups of HT patients using a logistic regression model was performed, adjusting for the same covariates as in the main case-control comparison.

Proportions of vitamin D deficiency $(<20 \mathrm{ng} / \mathrm{mL}$ ) were evaluated in all groups (all HT cases, MILD, OVERT and controls). We were also interested to compare the proportions of individuals with vitamin D deficiency between HT cases (all HT cases, MILD and OVERT) with control participants using the $\chi 2$-test. However, for these analyses only HT cases that matched controls for the season of blood draw were selected. Proportions of individuals with vitamin D deficiency were compared between the two subgroups of HT patients, using all samples, because the recruitment of HT cases was uniformly distributed throughout the year.

The next important goal of our study was to examine correlations between vitamin D and clinical phenotypes in all HT patients and the two subgroups of HT patients, using the Spearman correlation test. We tested 10 clinical phenotypes: thyroid hormones (TSH, T3, T4, fT4), thyroid antibodies (TPOAb and $\mathrm{TgAb}$ ), thyroid volume, number of hypothyroidism symptoms, systolic and diastolic blood pressure. The Bonferroni corrected $p$-value of 0.005 $(0.05 / 10)$ was used as a significance threshold. Prior to correlation analyses, vitamin D levels were corrected for age, gender, BMI, smoking and seasonality of blood sampling, whereas all clinical phenotypes were corrected for LT4 therapy status (yes/no) in all HT patients and OVERT cases using the linear regression model. All statistical analyses were performed using SPSS (version 20) statistical software.

\section{Results}

No significant differences in median vitamin D levels were detected between: (1) HT patients and control participants; HT cases and controls that matched for the seasonality of blood sampling; (2) MILD cases and controls; MILD cases and controls that matched for the seasonality of blood sampling; (3) OVERT cases and controls; OVERT cases and controls that matched for the seasonality of blood sampling (Table 2). However, a nominally significant difference in vitamin D levels between MILD and OVERT subgroups of HT cases was observed (Table 2).

Table 2. Results of logistic regression for vitamin D levels between tested groups.

\begin{tabular}{ccccccc}
\hline Tested Group & N 1 & N 2 & Median 1 (Q1-Q3) & Median 2 (Q1-Q3) & OR (95\% CI) & $p$-Value \\
\hline HT vs. controls $^{a}$ & 461 & $175^{c}$ & $19.7(14.4-25.2)$ & $17.3(13.2-22.7)$ & $0.987(0.958-1.017)$ & 0.401 \\
HT vs. controls $^{b}$ & 303 & 175 & $17.1(13.2-22.2)$ & $17.3(13.2-22.7)$ & $0.983(0.954-1.014)$ & 0.277 \\
MILD vs. controls $^{\text {a }}$ & 240 & 175 & $20.7(14.9-25.8)$ & $17.3(13.2-22.7)$ & $1.005(0.970-1.041)$ & 0.788 \\
MILD vs. controls $^{\mathrm{b}}$ & 155 & 175 & $17.7(14.0-22.6)$ & $17.3(13.2-22.7)$ & $1.002(0.966-1.038)$ & 0.927 \\
OVERT vs. controls $^{\mathrm{a}}$ & 219 & 175 & $19(13.9-24.5)$ & $17.3(13.2-22.7)$ & $0.971(0.937-1.006)$ & 0.105 \\
OVERT vs. controls $^{\mathrm{b}}$ & 147 & 175 & $16.7(12.1-21.7)$ & $17.3(13.2-22.7)$ & $0.966(0.930-1.002)$ & 0.065 \\
MILD vs. OVERT $^{\mathrm{a}}$ & 240 & 219 & $20.7(14.9-25.8)$ & $19(13.9-24.5)$ & $1.038(1.005-1.071)$ & 0.023 \\
\hline
\end{tabular}

a adjusted for age, sex, bmi, smoking status and seasonality; ${ }^{\mathrm{b}}$ matched for the season of blood draw (winter-spring) and adjusted as for ${ }^{\mathrm{a}}$; ${ }^{c}$ data for seasonality is missing in one control sample; $p<0.05$ are shown in bold, Q1: first quartile, Q3: third quartile, OR ( $95 \%$ CI)—odds ratio with $95 \%$ confidence intervals.

The distribution of vitamin D levels for all HT cases, MILD, OVERT and controls over the four seasons of the year is shown in Figure 1. Vitamin D levels show an expected distribution with the lowest values in winter/spring and highest in summer/autumn. Proportions of individuals with vitamin D deficiency were high in all the examined groups throughout all the year: all HT cases (51.84\%), MILD (47.92\%), OVERT (56.16\%). Proportions of individuals with vitamin D deficiency in the groups of HT cases that matched the controls for the season of blood draw (winter-spring) are, expectedly, higher: all HT cases $(64.69 \%)$, MILD (60.64\%), OVERT (68.7\%) and controls (60.79\%). Proportions of individuals with vitamin D deficiency in the groups of HT cases for the summer-autumn are lower: all HT cases (18.94\%), MILD (12\%), OVERT (27.23\%). Results of the comparison 
of proportions of individuals with vitamin D deficiency between HT cases, MILD and OVERT that matched the controls for the season of a blood draw and control participants, and, in-between MILD and OVERT cases (for all the year) are presented in Table 3. There were no significant differences in proportions of vitamin D deficiency between all the investigated groups.

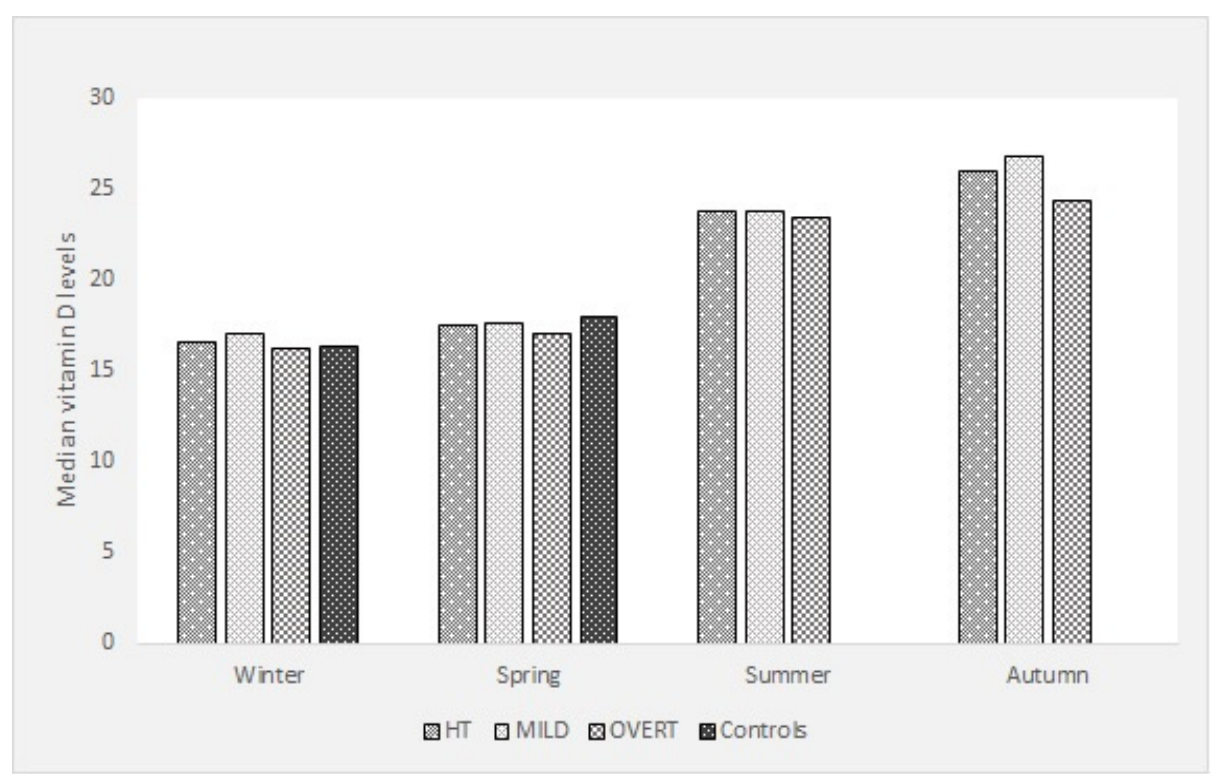

Figure 1. Seasonal distribution of vitamin D levels between groups of participants.

Table 3. Comparison of proportions of individuals with vitamin D deficiency.

\begin{tabular}{|c|c|c|c|c|c|}
\hline Tested Group & N 1 & N 2 & $\begin{array}{c}\text { Proportion } 1 \\
(\%)\end{array}$ & $\begin{array}{c}\text { Proportion } 2 \\
(\%)\end{array}$ & $\begin{array}{l}p \text {-Value } \\
(\chi 2 \text {-Test })\end{array}$ \\
\hline HT vs. controls ${ }^{a}$ & 196 & 107 & 64.69 & 60.79 & 0.394 \\
\hline MILD vs. controls ${ }^{a}$ & 94 & 107 & 60.64 & 60.79 & 0.977 \\
\hline OVERT vs. controls ${ }^{a}$ & 101 & 107 & 68.7 & 60.79 & 0.139 \\
\hline MILD vs. OVERT b & 115 & 123 & 47.92 & 56.16 & 0.077 \\
\hline
\end{tabular}

a matched for the season of blood draw of 6 months (winter-spring); ${ }^{\mathrm{b}}$ HT patients with vitamin D values for all the year.

Correlations between vitamin D levels and clinical phenotypes in all HT patients and the two subgroups of HT depending on severity (MILD and OVERT) are shown in Table 4. There were no Bonferroni corrected statistically significant correlations between vitamin $\mathrm{D}$ levels and clinical phenotypes, although two nominally significant correlations were identified $(p<0.05)$ : (1) a weak negative correlation between vitamin D and TSH in all HT patients $(\mathrm{r}=-0.113, p=0.029)$ and (2) a weak positive correlation between vitamin $\mathrm{D}$ and systolic blood pressure in OVERT HT patients $(r=0.205, p=0.025)$. 
Table 4. Correlation analyses between vitamin D levels and thyroid-related clinical phenotypes.

\begin{tabular}{|c|c|c|c|c|}
\hline Phenotype & & $\operatorname{ALL}(\mathrm{N}=461)$ & $\operatorname{MILD}(\mathrm{N}=240)$ & OVERT $(\mathrm{N}=219)$ \\
\hline \multirow{2}{*}{ TSH } & $\mathrm{r}$ & -0.113 & -0.092 & -0.015 \\
\hline & $p$ & 0.029 & 0.2 & 0.837 \\
\hline \multirow{2}{*}{ T3 } & $\mathrm{r}$ & -0.008 & -0.013 & -0.135 \\
\hline & $p$ & 0.882 & 0.857 & 0.07 \\
\hline \multirow{2}{*}{$\mathrm{T} 4$} & $\mathrm{r}$ & 0.026 & -0.025 & 0.053 \\
\hline & $p$ & 0.622 & 0.73 & 0.478 \\
\hline \multirow[b]{2}{*}{ fT4 } & $\mathrm{r}$ & 0.011 & -0.009 & -0.006 \\
\hline & $p$ & 0.832 & 0.906 & 0.941 \\
\hline \multirow{2}{*}{$\operatorname{TgAb}$} & $\mathrm{r}$ & -0.048 & -0.002 & -0.026 \\
\hline & $p$ & 0.357 & 0.979 & 0.724 \\
\hline \multirow{2}{*}{ TPOAb } & $\mathrm{r}$ & -0.039 & -0.058 & 0.047 \\
\hline & $p$ & 0.45 & 0.419 & 0.53 \\
\hline \multirow{2}{*}{ Thyroid volume } & $\mathrm{r}$ & -0.004 & 0.009 & 0.013 \\
\hline & $p$ & 0.947 & 0.899 & 0.867 \\
\hline \multirow{2}{*}{ No. of hypothyroid sym. } & $\mathrm{r}$ & 0.018 & 0.11 & -0.032 \\
\hline & $p$ & 0.755 & 0.178 & 0.693 \\
\hline \multirow{2}{*}{ Systolic blood pressure } & $\mathrm{r}$ & 0.073 & -0.03 & 0.205 \\
\hline & $p$ & 0.249 & 0.731 & 0.025 \\
\hline \multirow{2}{*}{ Diastolic blood pressure } & $\mathrm{r}$ & -0.001 & -0.04 & 0.112 \\
\hline & $p$ & 0.987 & 0.644 & 0.227 \\
\hline
\end{tabular}

r-Spearman correlation coefficient; nominally significant correlations are shown in bold.

\section{Discussion}

We performed a comprehensive study on associations between vitamin D levels and HT using a large group of deeply phenotyped HT cases and controls from the CROHT biobank. The main result of our cross-sectional and retrospective study is that we have not detected differences in vitamin $\mathrm{D}$ levels nor in proportions of vitamin $\mathrm{D}$ deficiency between all HT cases (HT patients of all disease stages) and controls. However, we detected a nominally significant difference in vitamin D levels between the MILD and OVERT subgroups of HT cases.

Our result of no association between vitamin D and HT is not supported by the recent systematic study and meta-analysis across 25 studies that reported a positive association of vitamin D levels and HT [29]. However, the same study concluded that many factors that influence patient heterogeneity, are frequently not included in analyses. The same conclusion was made by D'Aurizio et al. in a review paper from 2015, that pointed out that factors such as heterogeneity of the study population, seasonality of blood withdrawal, analytical variability of vitamin $\mathrm{D}$ assays and various definitions for declaring vitamin $\mathrm{D}$ deficiency may contribute to inconsistent findings [44]. Moreover, most of the studies that found a positive association between vitamin D levels and HT have had small numbers of participants with limited study designs [44]. For that reason, we performed the largest study to date including 461 clinically diagnosed HT patients and 176 clinically examined HT-free control participants from the same geographical region. The other advantage of our study, in comparison to the majority of other studies performed to date, is that we adjusted our analyses for the most comprehensive set of factors that are potential modifiers of individual vitamin D levels (age, sex, BMI, smoking status and season of blood sampling). In that way, we followed the most recent analytical recommendations to minimize the impact of other factors on vitamin D levels [29]. Our results of no association between vitamin D and HT are in line with several, albeit, smaller studies, the majority of which were not incorporated in the most recent meta-analysis [30-33].

To take the heterogeneity of the study population into account, we performed analyses using subsets of HT patients according to disease severity. We observed a nominally significant difference in vitamin D levels between MILD and OVERT subgroups of HT cases (Table 2) indicating that there may be subtle differences in vitamin D levels depending on disease severity. Our results suggest that HT patients from the OVERT group that are in 
advanced stages of disease (overt hypothyroidism and/or treated with LT4 therapy) have lower vitamin D levels (vitamin D median $=19 \mathrm{ng} / \mathrm{mL}$ ) and a higher proportion of vitamin D deficiency $(56.16 \%$ ) than those in the MILD group (vitamin D median $=20.7 \mathrm{ng} / \mathrm{mL}$; vitamin D deficiency $(47.92 \%)$ ) that are at the beginning of disease with preserved thyroid function (euthyroid/subclinical hypothyroid HT patients). Our results also suggest that it is vital to differentiate HT patients according to the stages of disease when performing analyses, as these subpopulations of patients have a different underlying physiology.

The main criteria for the differentiation of our patients into the OVERT group were $\mathrm{TSH}>10 \mathrm{mIU} / \mathrm{L}$ or the need for LT4 therapy intake, both of which reflect the malfunction of the thyroid gland. Although the two subgroups of patients have similar median TSH and thyroid hormone values (Table 1), the thyroid gland of patients from the MILD group was still capable of producing sufficient levels of thyroid hormones, whereas the thyroid gland of patients from the OVERT group was no longer functioning adequately and euthyroidism was restored due to synthetic hormone intake (LT4 therapy). There are other clinical features (Table 1) that point out that patients from the OVERT group, in comparison to patients from the MILD group, are in a progressed and more severe stage of HT. They are older and have higher median values for BMI, thyroid antibodies and the number of hypothyroidism symptoms. Taken altogether, these groups consist of two different entities of patients, which reflect the stage (severity) of HT.

The potential downside of our study is that our controls were recruited from December till June (winter-spring) which is the time of the year when vitamin D levels are low, which subsequently means that we do not have data for controls in the other half of the year, including the vitamin D peak in August. Although we have adjusted our analyses for seasonality, and additionally performed a subanalysis using HT cases that were tightly matched to controls on the date of recruitment (winter-spring), an interesting question may arise, and that is how fast vitamin D levels are restored in HT patients in comparison to controls during the sunny part of the year? It may be possible that HT patients in progressed stages of disease (OVERT) may not restore vitamin D values as efficiently as controls or patients at the beginning of disease. The rationale for this statement lies in our observation that controls and HT patients at the beginning of the disease (MILD) have higher vitamin $\mathrm{D}$ values, and lower proportions of vitamin $\mathrm{D}$ deficiency, than patients in more severe stages of disease (OVERT). Additionally, OVERT HT patients have the highest overall proportion of vitamin D deficiency among all the investigated groups of participants. We believe that future investigations should aim to investigate differences in vitamin D levels between a large cohort of HT patients in advanced stages of disease and healthy individuals, with vitamin D measures equally distributed throughout all the year, to estimate if lower vitamin D values are associated with progressed HT.

We also observed that vitamin D deficiency is high and common for HT patients and control participants, especially in the winter-spring season, which may mask potential significant differences in proportions of vitamin D deficiency between groups: all HT cases $(64.69 \%)$, MILD $(60.64 \%)$, OVERT $(68.7 \%)$ and controls $(60.79 \%)$. This is in line with Ferrari et al. who showed that $60-90 \%$ of the European population have deficient or insufficient vitamin D levels during "vitamin D winter" (November-March) [39]. We see that HT patients from the MILD group have similar levels of vitamin D deficiency as control individuals, whereas the highest proportion of vitamin D deficiency is in the OVERT group. Although our participants live in a region with many sunny days per year, it seems that other factors play a greater role in defining vitamin D levels, probably the most important one being indoor or office work. During the vitamin D peak season (summer-autumn) the proportion of vitamin D deficiency is considerably lower in all HT cases $(18.94 \%)$, MILD (12\%) and OVERT (27.23\%). This study recapitulates once more that there is a growing need of promoting outdoor activities for health purposes and/or the need for the intake of vitamin D supplementation. Although, we do not have information on daily sunlight exposure or outdoor activities of our participants, it is important to state that the region of Split, where our participants come from, has a Mediterranean climate 
with annual sunshine of more than $2600 \mathrm{~h}$ or the equivalent of 108 days. Our results, just like the results of Katrinaki et al. who also observed high vitamin D deficiency in another Mediterranean cohort, opens a question of redefining reference vitamin D values [45].

The next important observation of our study is a nominally significant weak negative correlation between vitamin D levels and TSH in all HT patients (Table 4). The majority of studies observed significant associations between vitamin D deficiency and TSH values in HT patients or weak negative correlations between vitamin D levels and TSH [28,46-48]. The most similar study to ours, by sample size and result, was reported by Kim et al. [47] who observed a significant negative correlation between vitamin D and serum TSH in the group of 369 AITD patients and 407 controls after adjustment for sample season, age, sex, and BMI $(\mathrm{r}=-0.127, p=0.013)$. Chao et al. [28] also observed a negative correlation and a similar effect to ours between vitamin D and TSH in 5230 participants, of which 373 were HT cases.

Similar results of a negative correlation between vitamin D and TSH were found in healthy populations as well [49-52]. However, one of the biggest studies, a cross-sectional analysis of 8042 individuals, did not observe a correlation between vitamin D and TSH, adjusting for age, sex and seasonal variation [45]. Taken altogether, the majority of literature records on this topic, including our study, suggest that HT patients perform physiologically similarly to healthy individuals in that they have a weak increase of TSH production associated with a decrease of vitamin D. A replication of our results in a greater sample of HT patients is needed for reaching a definite conclusion.

The second, nominally significant, weak positive correlation we observed was between vitamin D and systolic blood pressure in OVERT HT patients (Table 4). Most of the studies that investigated the influence of vitamin D on blood pressure in healthy individuals found an opposite effect, where higher vitamin D levels are correlated with lower blood pressure [53,54]. However, vitamin D deficiency has been associated with hypertension [54], and importantly, our OVERT group has a very high proportion of vitamin D deficiency, which could provide an explanation for our finding. The observed positive correlation is weak and the median systolic blood pressure in our OVERT group is not increased $(120 \mathrm{mmHg})$, therefore, it is not clear if the observed correlation in HT patients with overt hypothyreosis has clinical relevance.

A limitation of our study is that our control group is of a modest size, however the advantage is that all our controls are clinically confirmed HT-free individuals. Another limitation is that the observed correlations are weak and nominally significant. For that reason, our results need to be taken with precaution and replicated in a bigger sample. However, the advantage of our study is that we analyzed vitamin D with the most comprehensive list of clinical phenotypes in one of the biggest cohorts of HT patients to date.

\section{Conclusions}

In conclusion, we have not found an association between vitamin D levels and HT patients of all disease stages; however, results of our study indicate that there may be a subtle decrease in vitamin D levels associated with disease severity. We also observed high proportions of vitamin D deficiency in all study participants, alerting of necessity for outdoor activities for health purposes and/or the need for intake of vitamin D supplementation, especially for HT patients with a more severe form of disease. Our study shows that there is a need for a more detailed categorization of patients according to disease severity when performing scientific research.

Author Contributions: V.B.P. and M.C. conceived the study idea; D.K., A.B., M.V., I.G., A.P. and V.B.P. formed the biobank of patients with Hashimoto's thyroiditis (CROHT); M.C., A.B., M.V., and V.T.L. performed the measurements and diagnosis of HT; D.K. performed the statistical analysis; M.C., D.K. and V.B.P. drafted the manuscript; V.Š. and A.P. reviewed the manuscript. All authors have read and agreed to the published version of the manuscript. 
Funding: The formation of the CROHT biobank was supported by the Croatian Science Foundation under the project "Genome-wide association analysis of Hashimoto's thyroiditis" (grant no. 4950).

Institutional Review Board Statement: Ethics Committees from the University of Split School of Medicine (Classification no. 003-08/14-03/0001 and Registry no. 2181-198-03-04-14-0028; Classification no. 003-08/19-03/0003 and Reg. no. 2181-198-03-04-19-0019) and the University Hospital of Split (Classification no. 530-02/13-01/11; Registry no. 2181-147-01/06/J.B.-14-2; Classification no. 500-03/18-01/80 and Reg. no. 2181-147-01/06/M.S.-18-2) approved performance of this research and declared that the study is in accordance with the provisions of the Code of Ethics and the Helsinki Declaration.

Informed Consent Statement: All study participants were informed about planned research activities through the Information leaflet and they all signed an Agreement for participation prior to inclusion in the study.

Data Availability Statement: The data that support the findings of this study are available on reasonable request from the corresponding author.

Conflicts of Interest: There is no conflict of interest that could be perceived as prejudicing the impartiality of the research reported.

\section{References}

1. Nettore, I.C.; Albano, L.; Ungaro, P.; Colao, A.; Macchia, P.E. Sunshine vitamin and thyroid. Rev. Endocr. Metab. Disord. 2017, 18, 347-354. [CrossRef]

2. Sassi, F.; Tamone, C.; D'Amelio, P. Vitamin D: Nutrient, Hormone, and Immunomodulator. Nutrients 2018, 10, 1656. [CrossRef]

3. Muscogiuri, G.; Tirabassi, G.; Bizzaro, G.; Orio, F.; Paschou, S.A.; Vryonidou, A.; Balercia, G.; Shoenfeld, Y.; Colao, A. Vitamin D and thyroid disease: To D or not to D? Eur. J. Clin. Nutr. 2015, 69, 291-296. [CrossRef]

4. Mele, C.; Caputo, M.; Bisceglia, A.; Samà, M.T.; Zavattaro, M.; Aimaretti, G.; Pagano, L.; Prodam, F.; Marzullo, P. Immunomodulatory Effects of Vitamin D in Thyroid Diseases. Nutrients 2020, 12, 1444. [CrossRef] [PubMed]

5. Zhao, R.; Zhang, W.; Ma, C.; Zhao, Y.; Xiong, R.; Wang, H.; Chen, W.; Guo Zheng, S. Immunomodulatory Function of Vitamin D and Its Role in Auto-immune Thyroid Disease. Front. Immunol. 2021, 12, 352.

6. Vondra, K.; Stárka, L.; Hampl, R. Vitamin D and Thyroid Diseases. Physiol. Res. 2015, 64, S95-S100. [CrossRef]

7. Gallo, D.; Mortara, L.; Gariboldi, M.B.; Cattaneo, S.A.M.; Rosetti, S.; Gentile, L.; Noonan, D.M.; Premoli, P.; Cusini, C.; Tanda, M.L.; et al. Immunomodulatory effect of vitamin $\mathrm{D}$ and its potential role in the prevention and treatment of thyroid autoimmunity: A narrative review. J. Endocrinol. Investig. 2019, 43, 413-429. [CrossRef] [PubMed]

8. Kowalówka, M.; Główka, A.K.; Karaźniewicz-Łada, M.; Kosewski, G. Clinical Significance of Analysis of Vitamin D Status in Various Diseases. Nutrients 2020, 12, 2788. [CrossRef]

9. Pilz, S.; Zittermann, A.; Trummer, C.; Theiler-Schwetz, V.; Lerchbaum, E.; Keppel, M.H.; Grübler, M.R.; März, W.; Pandis, M. Vitamin D testing and treatment: A narrative review of current evidence. Endocr. Connect. 2019, 8, R27-R43. [CrossRef] [PubMed]

10. Holick, M.; Binkley, N.C.; Bischoff-Ferrari, H.; Gordon, C.M.; Hanley, D.A.; Heaney, R.P.; Murad, M.H.; Weaver, C.M. Evaluation, Treatment, and Prevention of Vitamin D Deficiency: An Endocrine Society Clinical Practice Guideline. J. Clin. Endocrinol. Metab. 2011, 96, 1911-1930. [CrossRef]

11. Adams, J.S.; Hewison, M. Update in vitamin D. J. Clin. Endocrinol. Metab. 2010, 95, 471-478. [CrossRef]

12. Shin, D.Y.; Kim, K.J.; Kim, D.; Hwang, S.; Lee, E.J. Low Serum Vitamin D Is Associated with Anti-Thyroid Peroxidase Antibody in Autoimmune Thyroiditis. Yonsei Med. J. 2014, 55, 476-481. [CrossRef]

13. Cashman, K.D.; Dowling, K.G.; Škrabáková, Z.; Gonzalez-Gross, M.; Valtueña, J.; De Henauw, S.; Moreno, L.; Damsgaard, C.T.; Michaelsen, K.F.; Mølgaard, C.; et al. Vitamin D deficiency in Europe: Pandemic? Am. J. Clin. Nutr. 2016, 103, 1033-1044. [CrossRef] [PubMed]

14. Caturegli, P.; De Remigis, A.; Rose, N. Hashimoto thyroiditis: Clinical and diagnostic criteria. Autoimmun. Rev. 2014, 13, 391-397. [CrossRef] [PubMed]

15. McLeod, D.S.A.; Cooper, D.S. The incidence and prevalence of thyroid autoimmunity. Endocrine 2012, 42, 252-265. [CrossRef] [PubMed]

16. Merrill, S.J.; Mu, Y. Thyroid autoimmunity as a window to autoimmunity: An explanation for sex differences in the prevalence of thyroid autoimmunity. J. Theor. Biol. 2015, 375, 95-100. [CrossRef] [PubMed]

17. Hiromatsu, Y.; Satoh, H.; Amino, N. Hashimoto's Thyroiditis: History and Future Outlook. Hormones 2013, 12, 12-18. [CrossRef] [PubMed]

18. Zaletel, K.; Gaberšček, S. Hashimoto's Thyroiditis: From Genes to the Disease. Curr. Genom. 2011, 12, 576-588. [CrossRef]

19. Brix, T.H.; Hegedüs, L. Twin studies as a model for exploring the aetiology of autoimmune thyroid disease. Clin. Endocrinol. 2012, 76, 457-464. [CrossRef]

20. Kolypetri, P.; King, J.; Larijani, M.; Carayanniotis, G. Genes and Environment as Predisposing Factors in Autoimmunity: Acceleration of Spontaneous Thyroiditis by Dietary Iodide in NOD.H2(h4) Mice. Int. Rev. Immunol. 2015, 34, 542-556. [CrossRef] 
21. Wu, L.; Yu, J.-C.; Kang, W.-M.; Ma, Z.-Q. Iodine nutrition and thyroid diseases. Zhongguo Yi Xue Ke Xue Yuan Xue Bao 2013, 35, $1-8$.

22. Desailloud, R.; Hober, D. Viruses and thyroiditis: An update. Virol. J. 2009, 6, 5. [CrossRef]

23. Burek, C.L.; Talor, M.V. Environmental triggers of autoimmune thyroiditis. J. Autoimmun. 2009, 33, 183-189. [CrossRef]

24. Liontiris, M.; Mazokopakis, E. A concise review of Hashimoto thyroiditis (HT) and the importance of iodine, selenium, vitamin D and gluten on the autoimmunity and dietary management of HT patients: Points that need more investigation. Hell. J. Nucl. Med. 2017, 20, 51-56. [CrossRef] [PubMed]

25. Tamer, G.; Arik, S.; Tamer, I.; Coksert, D. Relative vitamin D insufficiency in Hashimoto's thyroiditis. Thyroid 2011, 21, 891-896. [CrossRef]

26. Ventura, M.; Melo, M.; Carrilho, F. Selenium and Thyroid Disease: From Pathophysiology to Treatment. Int. J. Endocrinol. 2017, 2017, 1297658. [CrossRef] [PubMed]

27. Goswami, R.; Marwaha, R.K.; Gupta, N.; Tandon, N.; Sreenivas, V.; Tomar, N.; Ray, D.; Kanwar, R.; Agarwal, R. Prevalence of vitamin D deficiency and its relationship with thyroid autoimmunity in Asian Indians: A community-based survey. Br. J. Nutr. 2009, 102, 382-386. [CrossRef]

28. Chao, G.; Zhu, Y.; Fang, L. Correlation Between Hashimoto's Thyroiditis-Related Thyroid Hormone Levels and 25Hydroxyvitamin D. Front. Endocrinol. 2020, 11, 4. [CrossRef]

29. Stefanić, M.; Tokić, S. Serum 25-hydoxyvitamin D concentrations in relation to Hashimoto's thyroiditis: A systematic review, meta-analysis and meta-regression of observational studies. Eur. J. Nutr. 2020, 59, 859-872. [CrossRef] [PubMed]

30. Botelho, I.M.B.; Neto, A.M.; Silva, C.A.; Tambascia, M.A.; Alegre, S.M.; Zantut-Wittmann, D.E. Vitamin D in Hashimoto's thyroiditis and its relationship with thyroid function and inflammatory status. Endocr. J. 2018, 65, 1029-1037. [CrossRef]

31. Effraimidis, G.; Badenhoop, K.; Tijssen, J.G.P.; Wiersinga, W.M. Vitamin D deficiency is not associated with early stages of thyroid autoimmunity. Eur. J. Endocrinol. 2012, 167, 43-48. [CrossRef]

32. Musa, I.R.; Gasim, G.I.; Khan, S.; Ibrahim, I.A.; Abo-Alazm, H.; Adam, I. No Association between 25 (OH) Vitamin D Level and Hypothyroidism among Females. Open Access Maced. J. Med. Sci. 2017, 5, 126-130. [CrossRef]

33. Yasmeh, J.; Farpour, F.; Rizzo, V.; Kheradnam, S.; Sachmechi, I. Hashimoto Thyroiditis not Associated with Vitamin D Deficiency. Endocr. Pract. 2016, 22, 809-813. [CrossRef]

34. Brčić, L.; Barić, A.; Gračan, S.; Brekalo, M.; Kaličanin, D.; Gunjača, I.; Torlak Lovrić, V.; Tokić, S.; Radman, M.; Škrabić, V.; et al. Genome-wide association analysis suggests novel loci for Hashimoto's thyroiditis. J. Endocrinol. Investig. 2018, 42, 567-576. [CrossRef]

35. Brčić, L.; Barić, A.; Benzon, B.; Brekalo, M.; Gračan, S.; Kaličanin, D.; Škrabić, V.; Zemunik, T.; Barbalić, M.; Novak, I.; et al. AATF and SMARCA2 are associated with thyroid volume in Hashimoto's thyroiditis patients. Sci. Rep. 2020, 10, 1754. [CrossRef]

36. Ovesen, L.; Andersen, R.; Jakobsen, J. Geographical differences in vitamin D status, with particular reference to European coun-tries. Proc. Nutr. Soc. 2003, 62, 813-821. [CrossRef] [PubMed]

37. Zhu, X.-W.; Liu, K.-Q.; Wang, P.-Y.; Liu, J.-Q.; Chen, J.-Y.; Xu, X.-J.; Xu, J.-J.; Qiu, M.-C.; Sun, Y.; Liu, C.; et al. Cohort profile: The Westlake BioBank for Chinese (WBBC) pilot project. BMJ Open 2021, 11, e045564. [CrossRef]

38. Tirabassi, G.; Cutini, M.; Salvio, G.; Cerqueni, G.; Lenzi, A.; Balercia, G. Influence of vitamin D levels on the cardiovascular profile of hypogonadal men. J. Endocrinol. Investig. 2017, 40, 1007-1014. [CrossRef] [PubMed]

39. Ferrari, D.; Lombardi, G.; Strollo, M.; Pontillo, M.; Motta, A.; Locatelli, M. Association between solar ultraviolet doses and vitamin D clinical routine data in European mid-latitude population between 2006 and 2018. Photochem. Photobiol. Sci. 2019, 18, 2696-2706. [CrossRef] [PubMed]

40. Cai, Z.; Zhang, Q.; Xia, Z.; Zheng, S.; Zeng, L.; Han, L.; Yan, J.; Ke, P.; Zhuang, J.; Wu, X.; et al. Determination of serum 25-hydroxyvitamin D status among population in southern China by a high accuracy LC-MS/MS method traced to reference measurement procedure. Nutr. Metab. 2020, 17, 8-13. [CrossRef] [PubMed]

41. Won, J.W.; Jung, S.K.; Jung, I.A.; Lee, Y. Seasonal Changes in Vitamin D Levels of Healthy Children in Mid-Latitude, Asian Urban Area. Pediatr. Gastroenterol. Hepatol. Nutr. 2021, 24, 207-217. [CrossRef] [PubMed]

42. Pearce, S.; Brabant, G.; Duntas, L.; Monzani, F.; Peeters, R.P.; Razvi, S.; Wemeau, J.-L. 2013 ETA Guideline: Management of Subclinical Hypothyroidism. Eur. Thyroid. J. 2013, 2, 215-228. [CrossRef]

43. Brunn, J.; Block, U.; Ruf, G.; Bos, I.; Kunze, W.P.; Scriba, P.C. Volumetric analysis of thyroid lobes by real-time ultrasound. Dtsch. Med. Wochenschr. 1981, 106, 1338-1340. [CrossRef] [PubMed]

44. D'Aurizio, F.; Villalta, D.; Metus, P.; Doretto, P.; Tozzoli, R. Is vitamin D a player or not in the pathophysiology of autoimmune thyroid diseases? Autoimmun. Rev. 2015, 14, 363-369. [CrossRef] [PubMed]

45. Katrinaki, M.; Kampa, M.; Margioris, A.; Castanas, E.; Malliaraki, N. Vitamin D levels in a large Mediterranean cohort: Reconsidering normal cut-off values. Hormones 2016, 15, 205-223. [CrossRef] [PubMed]

46. Evliyaoğlu, O.; Acar, M.; Özcabı, B.; Erginöz, E.; Bucak, F.; Ercan, O.; Kucur, M. Vitamin D Deficiency and Hashimoto's Thyroiditis in Children and Adolescents: A Critical Vitamin D Level for This Association? J. Clin. Res. Pediatr. Endocrinol. 2015, 7, 128-133. [CrossRef] [PubMed]

47. Kim, D. Low vitamin D status is associated with hypothyroid Hashimoto's thyroiditis. Hormones 2016, 15, 385-393. [CrossRef]

48. Kivity, S.; Agmon-Levin, N.; Zisappl, M.; Shapira, Y.; Nagy, E.V.; Dankó, K.; Szekanecz, Z.; Langevitz, P.; Shoenfeld, Y. Vitamin D and autoimmune thyroid diseases. Cell. Mol. Immunol. 2011, 8, 243-247. [CrossRef] [PubMed] 
49. Chailurkit, L.-O.; Aekplakorn, W.; Ongphiphadhanakul, B. High Vitamin D Status in Younger Individuals Is Associated with Low Circulating Thyrotropin. Thyroid 2013, 23, 25-30. [CrossRef]

50. Zhang, Q.; Wang, Z.; Sun, M.; Cao, M.; Zhu, Z.; Fu, Q.; Gao, Y.; Mao, J.; Li, Y.; Shi, Y.; et al. Association of high vitamin d status with low circulating thyroid-stimulating hormone independent of thyroid hormone levels in middle-aged and elderly males. Int. J. Endocrinol. 2014, 631819, 16. [CrossRef]

51. Barchetta, I.; Baroni, M.G.; Leonetti, F.; De Bernardinis, M.; Bertoccini, L.; Fontana, M.; Mazzei, E.; Fraioli, A.; Cavallo, M.G. TSH levels are associated with vitamin D status and seasonality in an adult population of euthyroid adults. Clin. Exp. Med. 2014, 15, 389-396. [CrossRef] [PubMed]

52. Das, G.; Taylor, P.N.; Javaid, H.; Tennant, B.P.; Geen, J.; Aldridge, A.; Okosieme, O. Seasonal Variation of Vitamin D and Serum Thyrotropin Levels and Its Relationship in a Euthyroid Caucasian Population. Endocr. Pract. 2018, 24, 53-59. [CrossRef] [PubMed]

53. Fonseca Valle, D.; Giannini, D.T. Correlation between vitamin D and blood pressure in adolescents. Int. J. Adolesc. Med. Health 2019, 32, 20170165. [CrossRef]

54. Lin, L.; Zhang, L.; Li, C.; Gai, Z.; Li, Y. Vitamin D and Vitamin D Receptor: New Insights in the Treatment of Hypertension. Curr. Protein Pept. Sci. 2019, 20, 984-995. [CrossRef] [PubMed] 